\title{
Phenotypic Detection and Occurrence of Extended-Spectrum Beta-Lactamases in Clinical Isolates of Klebsiella pneumoniae and Escherichia coli at a Tertiary Hospital in Trinidad \& Tobago
}

\author{
Patrick E. Akpaka and William H. Swanston \\ Department of Para-Clinical Sciences, Faculty of Medical Sciences, The University of the West Indies, St. Augustine; Trinidad \& Tobago
}

\begin{abstract}
The incidence and distribution of ESBL producing microorganisms such as $E$. coli and $K$. pneumoniae have been demonstrated and varies in different health care facilities and as well as other countries This study was carried out to determine the frequency of occurrence and the antimicrobial susceptibility pattern of ESBL producing $E$. coli and K. pneumoniae species from clinical isolates at a tertiary hospital in Trinidad \& Tobago. Standard microbiological procedures and automated MicroScan System was used to identify, screen for putative ESBL production and determine antimicrobial susceptibility of 1,118 clinical isolates of Enterobacteriaceae species at the microbiology laboratory of the Eric Williams Medical Science Complex, Trinidad \& Tobago over a 36 months period. All ESBL producing isolates flagged by the automated system were further confirmed by E-test method. The E-test confirmed a $15.2 \%$ ESBL rate among the $K$. pneumoniae isolates and $9.3 \%$ among the $E$. coli isolates. There was also a $1.8 \%$ rate of ESBL production in $K$. pneumoniae and $0.2 \%$ in $E$. coli isolates from specimens received from community health facilities into the laboratory. Isolates recovered from the intensive care unit of the hospital had $2.1 \% \mathrm{E}$. coli and 8.2\% K. pneumoniae ESBL producers. Although all ESBL positive isolates were completely susceptible to imipenem and meropenem; and all positive $K$. pneumoniae isolates were susceptible to amikacin, there was a low susceptibility of ESBL positive $E$. coli to the aminoglycosides. However, susceptibility of these ESBL producing isolates to the fluoroquinolones varied. There is a high rate of ESBL production among isolates of $E$. coli and $K$. pneumoniae at this hospital that is linked to the extensive inappropriate use of third generation cephalosporins in the country. Further molecular studies are needed to characterize the types of these ESBL prevailing in the country.

Key-Words: ESBL detection, E-test methods, E. coli, K. pneumoniae, antimicrobial susceptibility, Trinidad \& Tobago, Tertiary hospital.
\end{abstract}

Extended-spectrum $\beta$-lactamases (ESBL) are enzymes that hydrolyze broad-spectrum cephalosporins and monobactams including oxyimino-cephalosporins, as well as older $\beta$-lactams antimicrobial agents which are inhibited by clavulanic acids. Since these enzymes were initially recognized in Klebsiella pneumoniae in Germany in 1983, they have been increasingly described worldwide [1-5]. The incidence and distribution of ESBL producing microorganisms such as $E$. coli and $K$. pneumoniae have been demonstrated and varies in different health care facilities and as well as other countries $[6,7]$.

In Trinidad and Tobago, the first report of incidence of ESBL was in a neutropenic patient at the Port of Spain General Hospital in an isolate of Salmonella enteritidis, which exhibited resistance to all penicillins and cephalosporins (including third generation cephalosporins), aminoglycosides and trimethoprim-sulphamethazole [8-10]. The several worldwide report of high prevalence rates and outbreaks of ESBL-producing microorganisms underscores the importance of searching and detecting their occurrence in all hospitals offering ambulatory services.

Given the difficulty in detecting ESBL production as well as inconsistencies in reporting [11], the prevalence of ESBL will most likely be underestimated in the developing countries.

Received on 6 April 2008; revised 5 November 2008.

Address for correspondence: Dr. Patrick. E. Akpaka. Department of Para-Clinical Sciences, Faculty of Medical Sciences, The University of the West Indies, St. Augustine, Trinidad \& Tobago. Email: peakpaka@yahoo.co.uk. Phone (868) 736-0440; Fax: (868) 663-3797.

The Brazilian Journal of Infectious Diseases

2008;12(6):516-520. (C) 2008 by The Brazilian Journal of Infectious Diseases and Contexto Publishing. All rights reserved.
Infections by these microorganisms producing ESBL do pose therapeutic dilemma and challenging infection control problems in many hospitals. Therefore, this present study was conducted to determine the prevalence and antimicrobial susceptibilities among clinical isolates of ESBL producing $E$. coli and $K$. pneumoniae at one of the regional tertiary hospitals in the country.

\section{Material and Methods}

Bacterial Isolates

Consecutive non-duplicate clinical Klebsiella pneumoniae and Escherichia coli isolates recovered from specimens processed at the microbiology laboratory of the Eric Williams Medical Sciences Complex (EWMSC) were used. The EWMSC is a tertiary ambulatory hospital in Trinidad \& Tobago, a twoisland country located in the southern Caribbean Sea, off the coast of Venezuela. The study was done over a 3-year period, between December 2004 and November 2007.

\section{MicroScan Analysis}

Automated micro dilution MicroScan WalkAway-96 System (Dade Behring) was used to perform the identification and antimicrobial susceptibility tests using Neg Combo 32 B1017-302 and Neg Combo 35 B1017-305 panels. All procedures were performed according to the manufacturer's instructions. The integrated LabPro 2.0 version that includes the Alert expert system uses growth in the presence of ceftazidime $(1 \mu \mathrm{g} / \mathrm{mL})$ and cefotaxime $(4 \mu \mathrm{g} / \mathrm{mL})$ at concentrations recommended by the CLSI for ESBL screening [12]. The MicroScan expert system software does not permit identification of ESBL producers among Enterobacteriaceae 
organisms other than the E. coli and Klebsiella species. In addition, the system in our laboratory only alerts the user to the possibility of ESBL producers.

MICs obtained for several antimicrobial agents including cefotaxime, ceftriaxone, ceftazidime and aztreonam from the WalkAway system were interpreted according to CLSI breakpoints [12]. All isolates of E. coli and Klebsiella pneumoniae primarily indicated by the MicroScan system as possible ESBL producers with MIC breakpoints interpreted as resistant or intermediate by the system were regarded as having decreased susceptibility for the purpose of this study. These isolates are stored at $-70^{\circ} \mathrm{C}$ in tripticase soy broth (Difco Laboratories, Detroit MI, USA) supplemented with 5\% glycerol until further testing. All isolates that were not alerted as ESBL producers by the MicroScan had their antimicrobial susceptibility patterns compared with the ESBL positive isolates.

\section{E-Test ESBL Confirmatory Tests}

E-test strips, cefotaxime CT having a gradient concentration of $0.25-16 \mu \mathrm{g} / \mathrm{mL}$ and ceftazidime TZ $(0.5-$ $32 \mu \mathrm{g} / \mathrm{mL}$ ) at one end; and cefotaxime plus clavulanic acid CTL $(0.016-1 \mu \mathrm{g} / \mathrm{mL}+4 \mu \mathrm{g} / \mathrm{mL})$ or ceftazidime with clavulanic acid TZL $(0.064-4 \mu \mathrm{g} / \mathrm{mL}+4 \mu \mathrm{g} / \mathrm{mL})$ at the other end were used in accordance with the protocols from the manufacturer [13]. ESBL production was determined if the microbial isolate had an $\mathrm{MIC} \mu \mathrm{g} / \mathrm{mL}$ of $\geq 0.5$ for $\mathrm{CT}, \geq 1$ for $\mathrm{TZ}$; and $\mathrm{MIC} \mu \mathrm{g} / \mathrm{mL}$ ratio of $\geq 8$ for CT/CTL or TZ/TZL. ESBL production was also identified by the presence of phantom zone or a deformation of the cefotaxime or ceftazidime ellipse. A result was considered indeterminate when the MICs were outside the range of the MICs of the respective E-test ESBL test strip, and a MIC ratio could therefore not be calculated. Control strain for all the phenotypic testing were $E$. coli ATCC 25922 (negative control) and K. pneumoniae ATCC 700603 (ESBL positive).

\section{Results}

During the study period, 1,118 isolates of K. pneumoniae (402) and E. coli (716) were recovered and identified by the MicroScan machine from clinical specimens processed at the microbiology laboratory of the hospital. Urinary tract isolates $67.2 \%$ (752); specifically $72.2 \% \mathrm{E}$. coli and $27.8 \% \mathrm{~K}$. pneumoniae were dominant. Isolates from soft tissues accounted for $23.6 \%$, blood $5.3 \%$ and respiratory tract $3.1 \%$. The performance of the automated MicroScan system and ESBL E-test in screening and confirming ESBL production in the E. coli and K. pneumoniae isolates is summarized in Table 1. The MicroScan putatively identified 275 isolates as ESBL producers comprising $15.2 \%$ K. pneumoniae and 9.4\% E. coli respectively.

The E-test method confirmed ESBL production in 9.3\% E. coli and $15.2 \% \mathrm{~K}$. pneumoniae of the 1,118 isolates as evidenced by ratios of the MIC of ceftazidime and/or cefotaxime plus clavulanic acid of $\geq 8$, as indicated by ESBL activity (Figures 1) according to E-test interpretative guidelines and CLSI criteria. The collective ESBL producing E. coli and $K$. pneumoniae isolates from specimens of patients coming from the community health facilities was 1.8\% (21/1118); and from the intensive care unit of the hospital $4.2 \%(48 / 1118)$ comprising E. coli 2.1\% (16/716) and K. pneumoniae $8.2 \%$ (33/402).

The antibiotic susceptibility patterns of the isolates confirmed as ESBL producers were analyzed and compared with non-ESBL producers (Table 2). All isolates producing ESBL were susceptible to the carbapenems (imipenem and meropenem). There was equally a high susceptibility rate of these isolates producing ESBL to cefotetan, 96.3\% to E. coli and $98 \%$ to $K$. pneumoniae. It should be noted that microorganisms producing ESBL enzymes are also often resistant to quinolones, aminoglycosides and other antimicrobials via other mechanisms.

Surprisingly, while all K. pneumoniae ESBL producers were fully susceptible to amikacin (100\%), only 45\% of E. coli was susceptible. There was low susceptibility of the E. coli producing ESBLs isolates to gentamicin (8.1\%), tobramycin (9.2\%) and trimethoprim/sulphamethazole (2.5\%), while susceptibility rates of K. pneumoniae isolates occurred $22.2 \%$ in tobramycin, 39.3\% gentamicin and 51.8\% in trimethoprim/ sulphamethazole.

As expected, there was complete resistance to the cephalosporins by the ESBL producing isolates. There was however slightly increased susceptibility of ESBL producing $E$. coli isolates to fluoroquinolones (ciprofloxacin $48.5 \%$, levofloxacin $61.6 \%$ and moxifloxacin $68.9 \%$ ). The $K$. pneumoniae isolates susceptibility to ciprofloxacin was $61.8 \%$, levofloxacin $65.5 \%$, and moxifloxacin $51.5 \%$.

\section{Discussion}

The phenotypic data generated in this study indicate that at the Eric Williams Medical Sciences Complex, a significantly large number of the enterobacteriaceae isolates produce ESBL at the rate of $15.2 \%$ in K. pneumoniae and 9.3 in E. coli. This rate of ESBL production in $E$. coli isolates observed in this study is higher than the rates in Latin America and Europe at $8.5 \%$ and $5.3 \%$ respectively [14]; but similar to the rate observed in Hong Kong and lower than $24.5 \%$ in mainland China [15]. The K. pneumoniae rate in this study is however lower than the rates reported by Winokur et al. from Latin America [14] as well as rates reported by Hirakata Y. et al. from Singapore, mainland China, South Africa and Philippines [15].

The single most reason for this high rate of ESBL production among these $K$. pneumoniae and E. coli isolates in this hospital is the extensive inappropriate use of cephalosporins in the country as reported by Pinto Pereira et al. [16]. This selective pressure created by the use of this third generation cephalosporins has also been described as one of the most important factors elsewhere $[17,18]$.

The specific $15.2 \%$ K. pneumoniae and 9.3\% E. coli ESBL production prevalence rate encountered in the present study was also higher than K. pneumoniae and E. coli rate of $9.5 \%$ 
Table 1. Results of MicroScan WalkAway system and E-test methods in detecting ESBL production from K. pneumoniae and E. coli isolates at Eric Williams Medical Sciences Complex Trinidad \& Tobago from December 2004 -August 2007.

\begin{tabular}{lcccccrr}
\hline Species & N & \multicolumn{2}{c}{ MicroScan (\%) } & N & \multicolumn{3}{c}{ E-test (\%) } \\
\cline { 3 - 8 } & & \multicolumn{1}{c}{ Pos } & Neg & & Pos & Neg & Ind* \\
\hline K. pneumoniae & 402 & $170(15.2)$ & 232 & 170 & $162(14.5)$ & 0 & $8(0.7)$ \\
E. coli & 716 & $105(9.4)$ & 611 & 105 & $99(8.9)$ & 2 & $4(0.4)$ \\
Total & 1,118 & 275 & 843 & 273 & 261 & 2 & 12 \\
\hline
\end{tabular}

$\mathrm{N}$ = number of isolates tested; Pos = positive; Neg = negative; Ind* = indeterminate, isolates observed to be indeterminate for ESBL production using the E-test method are regarded as positives for ESBL production.

Table 2. In vitro antibiotic susceptibility patterns of suspected or confirmed ESBL producing isolates of K. pneumoniae and E. coli recovered from clinical specimens processed at the microbiology laboratory of the Eric Williams Medical Sciences Complex Trinidad \& Tobago from December 2004 to August 2007.

\begin{tabular}{|c|c|c|c|c|}
\hline \multirow[t]{3}{*}{ Antimicrobial } & \multicolumn{4}{|c|}{ Percentage of susceptible isolates } \\
\hline & \multicolumn{2}{|c|}{ E. coli } & \multicolumn{2}{|c|}{ K. pneumoniae } \\
\hline & ESBL $^{-v e}$ & ESSBL $^{+v e}$ & ESBL $^{-v e}$ & ESBL $^{+v e}$ \\
\hline Ampicillin & 6.7 & 0 & 0 & 0 \\
\hline Amp/Sulbactam & 13.3 & 0 & 30 & 0 \\
\hline Piperacillin & 20 & 0 & 8.5 & 0 \\
\hline Pip/Tazo & 86.7 & 80 & 78 & 62.5 \\
\hline Aztreonam & 67 & 0 & 70 & 0 \\
\hline Meropenem & 100 & 100 & 100 & 100 \\
\hline Imipenem & 100 & 100 & 100 & 100 \\
\hline Ciprofloxacin & 75 & 48.5 & 75.5 & 61.8 \\
\hline Levofloxacin & 70 & 61.6 & 70 & 65.5 \\
\hline Moxifloxacin & 87 & 68.9 & 78.5 & 51.5 \\
\hline Cefotetan & 100 & 96.3 & 100 & 98 \\
\hline Cefuroxime & 40 & 0 & 53.8 & 0 \\
\hline Cefotaxime & 37 & 0 & 75 & 0 \\
\hline Ceftriaxone & 42 & 0 & 58.5 & 0 \\
\hline Ceftazidime & 18 & 0 & 30.5 & 0 \\
\hline Cefepime & 45 & 0 & 69.2 & 12.5 \\
\hline Amikacin & 86.7 & 45 & 75 & 100 \\
\hline Gentamicin & 40 & 8.1 & 77 & 39.3 \\
\hline Tobramycin & 47 & 9.2 & 68.8 & 22.2 \\
\hline Trimeth/Sulfa & 26.6 & 2.5 & 78.4 & 51.8 \\
\hline
\end{tabular}

and 2.9\% in 1998; and 6.3\% and 0.7\% in 2001 by Cherian BP et al. [10] in another tertiary hospital in the country. Our rate is also lower than $18.2 \%$ encountered in K. pneumoniae from Jamaica, another Caribbean country [19]. The reason for this variation between the results could largely be due to difference in study designs. This is evident in the studies done by Cherian et al. and Nicholson et al. [10,19] where less number of isolates, collected over a shorter period of time were was used.

Conversely, the frequency of ESBL producers from isolates from specimens of patients who attended the community health centers in this present study was lower than what was reported in India [20] but higher than $1.48 \%$ reported from Brazil [21]. The high rates of ESBL seen among isolates of patients treated in the community is not because of over the counter sales of antibiotics practiced in India [20] but mostly because of poorly directed therapy among our general practitioners in which there is indiscriminate practice of antibiotics prescription for patients with viral infections in the community in Trinidad [22]. Although the ESBL producing isolates from the intensive care hospital of our study $2.1 \%$ E. coli $2.1 \%$ and $8.2 \% \mathrm{~K}$. pneumoniae respectively, yet the rates are still lower than $11.2 \%$ in $E$. coli and $16.2 \%$ K. pneumoniae reported in USA [23] or $80 \%$ in K. pneumoniae isolates reported in India [24].

There is significantly high rate of resistance of all the $K$. pneumoniae isolates to the fluoroquinolones. This clearly demonstrates that these antimicrobial agents are to be used with caution in infections such as urinary tract infections or intra abdominal infections as has been documented elsewhere [25]. All isolates of E. coli and $K$. pneumoniae in ESBL phenotypes in this study were susceptible to the carbapenems (meropenem and imipenem). 
Figure 1. Detection of ESBL carriage with an E-test ESBL strips. Ceftazidime MIC against E. coli isolate in A is $>32 \mu \mathrm{g} / \mathrm{mL}$ in the absence of clavulanate and $0.125 \mu \mathrm{g} / \mathrm{mL}$ in the presence of clavulanate. Ceftazidime MIC against K. pneumoniae isolate in B is $>32 \mu \mathrm{g} / \mathrm{mL}$ in the absence of clavulanate and $0.125 \mu \mathrm{g} / \mathrm{mL}$ in the presence of clavulanate. Observe the phantom zone production in $\mathrm{B}$. As the ratio of ceftazidime with and without clavulanate is $\geq 8$, the isolates were phenotypically determined as ESBL producers.

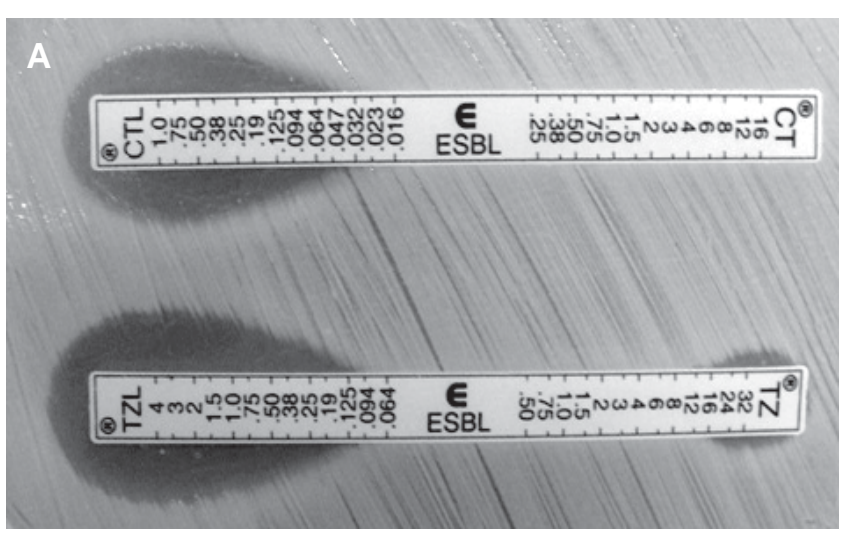

This result is in agreement with reports from Spain, North America and United Kingdom [26-28]. This augurs well in this hospital because these antimicrobials are licensed in the country, easily and readily available; and can be recommended as drug of choice for treatment of infections by these organisms. The main limitation of this study is the inability to characterize the types of ESBL prevailing in the country.

\section{Conclusion}

Despite the inability to characterize the ESBL types in this study, there is a high rate of ESBL among the $E$. coli and $K$. pneumoniae isolates encountered in this hospital that may be attributed to the extensive inappropriate use of third generation cephalosporins. There is a strong need to carry out further molecular studies to delineate the prevailing types of ESBL in this hospital and country.

\section{Acknowledgement}

Fund for this study was partly provided by the University of the West Indies, St. Augustine. Thanks to the staff of the Eric Williams Medical Sciences Complex and the Paraclinical Sciences Department of the Faculty of Medical Sciences, UWI for their invaluable assistance. Special thanks also to A. Akpaka fro proof reading and editing the manuscript.

\section{References}

1. Knothe H., Shah P., Krcmery V., et al. Transferable resistance to cefotaxime, cefoxitin, cefamandole and cefuroxime in clinical isolates of Klebsiella pneumoniae and Serratia marcescens. Infection 1983;11:315-7.

2. Kliebe C., Nies B.B.A., Meyer J.F., et al. Evolution of plasmidcoded resistance to broad-spectrum cephalosporins. Antimicrob Agents Chemother 1985;28:302-7.

3. Jacoby G.A., Medeiros A.A., O’Brien T.F., et al. Broad-spectrum transmissible $\beta$-lactamases. N. Engl J Med 1991;319:723-4.

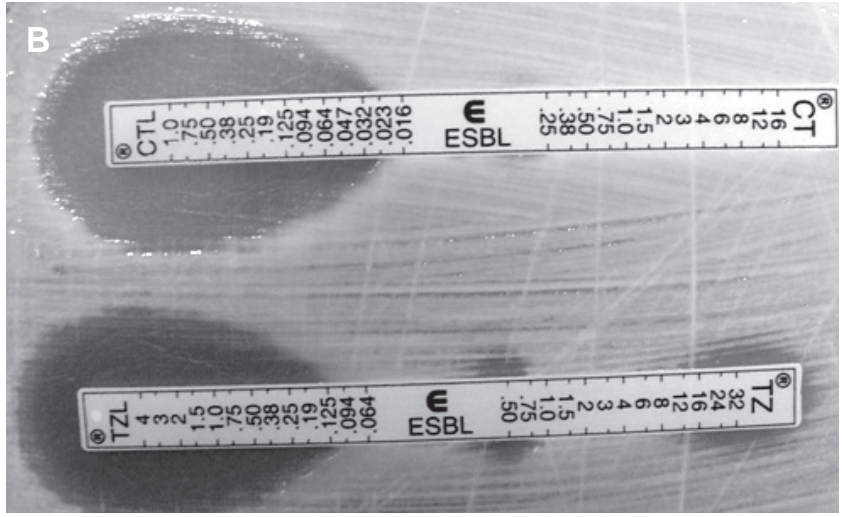

4. Pfaller M.A., Jones R.N. For the MYSTIC Study Group (Americas). MYSTIC (Meropenem Yearly Susceptibility Test Information Collection) results from the Americas: resistance implications in the treatment of serious infections. Journal of Antimicrobial Chemotherapy 2000;46:25-37.

5. Goossens H., For the MYSTIC Study Group (European Centres). MYSTIC (Meropenem Yearly Susceptibility Test Information Collection) results from Europe: comparison of antibiotic susceptibilities between countries and centre types. Journal of Antimicrobial Chemotherapy 2000;46:39-52.

6. Hernández J.R., Martínez-Martínez L., Cantón R., et al. National Study of Escherichia coli and Klebsiella pneumoniae producing Extended-Spectrum $\beta$-lactamases in Spain. Antimicrob Agents Chemother 2005;49:2122-5.

7. Edelstein M., Pimkin M., Palagin I., et al. Prevalence and Molecular Epidemiology of CTX-M Extended-Spectrum B-LactamaseProducing Escherichia coli and Klebsiella pneumoniae in Russian Hospitals. Antimicrob Agents Chemother 2003;47:3724-32.

8. Cherian B.P., Singh N., Charles W., Prabhakar P. Extended spectrum $\beta$-lactamase producing Salmonella enteritidis in Trinidad \& Tobago. Emerg Infect Dis 1999;5:181-2.

9. Prabhakar P., the Caribbean Antimicrobial Resistance Surveillance Group, Trinidad and Tobago. Antimicrobial resistance in the Caribbean. West Indian Med J 2000;49:62.

10. Cherian B.P., Manjunath M., Pereira L.M., Prabhakar P. Extended spectrum $\beta$-lactamase producing Enterobacteriaceae in a tertiary care hospital in Trinidad \& Tobago. West Indian Med J 2000;52:31-3.

11. Steward C.D., Wallace D., Hubert S.K., et al. Ability of laboratories to detect emerging antimicrobial resistance in nosocomial pathogens: a survey of project ICARE laboratories. Diagn Microbial Infect Dis 2000;38:59-67.

12. Clinical and Laboratory Standard Institute. Performance standards for antimicrobial susceptibility testing: sixteenth informational supplement. 2006 CLSI document M100-S16. CLSI, Wayne, Pa.

13. AB Biodisk E-test ${ }^{\circledR}$ ESBL Cefotaxime/Cefotaxime + Clavulanic acid and Ceftazidime/Ceftazidime + Clavulanic acid Package Insert for in vitro confirmation of ESBL. AB Biodisk, Solna, Sweden, 2005.

14. Winokur P.L., Canton R., Casellas J.M., Legakis N. Variations in the prevalence of strains expressing an Extended-spectrum $\beta$ lactamase phenotype and characterization of isolates from Europe, the Americas, and the Western Pacific Region. Clin Infect Dis 2001;32(Suppl 2):S94-S101. 
15. Hirakata Y., Matsuda J., Miyazaki Y., et al. SENTRY Asia-Pacific Participants. Regional variation in the prevalence of extendedspectrum beta-lactamase-producing clinical isolates in the Asia Pacific region (SENTRY 1998-2002). Diagn Microbiol Infect Dis 2005;52(4):323-9.

16. Pinto Pereira L.M., Phillips M., Ramlal H., et al. Third generation cephalosporin use in a tertiary hospital in Port of Spain, Trinidad: need for antibiotic policy. BMC Infect Dis 2004,4:59.doi 10.1186/1471-2334-4-59.

17. Philippon A., Arlet G., Lagrange P.H. Origin and impact of plasmidmediated extended-spectrum $\beta$-lactamases. Eur J Clin Microbiol Infect Dis 1994;13:S17-9.

18. Thomson K.S., Prevan A.M., Sander C.C. Novel Plasmid-mediated $\beta$-lactamases in Enterobacteriaceae: emerging problems for new B-lactam antibiotics. Curr Clin Topics Infect Dis 1996;16:151-63.

19. Nicholson A.M., Gayle P., Roye-Green K. Extended Spectrum Beta-Lactamase Producing organisms at the University Hospital of the West Indies. West Indian Med J 2004;53:104-8.

20. Gupta V., Datta P. Extended-spectrum beta-lactamases in community isolates from North India: frequency and predisposing factors. Int $J$ Infect Dis $2007 ; 11: 88-9$.

21. Minarini L.A., Gales A.C., Palazzo I.C., Darini A.L. Prevalence of Community-occurring Extended Spectrum beta-LactamaseProducing Enterobacteriaceae in Brazil. Curr Microbiol 2007;54(5):335-41.
22. Mohan S., Dharamraj K., Dindial R., et al. Physician behaviour for antimicrobial prescribing for paediatric upper respiratory tract infections: a survey in general practice in Trinidad, West Indies. Ann Clin Microbiol Antimicrob 2004;143:11.

23. Streit J.M., Jones R.N., Sader H.S., Fritsche T.R. Assessment of pathogen occurrences and resistance profiles among infected patients in the intensive care unit: report from the SENTRY Antimicrobial Surveillance Program (North America, 2001). Int J Antimicrob Agents 2004;24(2):111-8.

24. Mathur P., Kapil A., Das B., Dhawan B. Prevalence of extended spectrum beta lactamase producing Gram-negative bacteria in a tertiary care hospital. Indian J Med Res 2002;115:153-7.

25. Fridkin S.K., Steward C.D., Edwards J.R. Surveillance of antimicrobial use and antimicrobial resistance in United States hospitals: project ICARE phase 2. Clin Infect Dis 1999;29:245-52.

26. Pascual A., Perea E., Alvarez M., et al. The Meropenem Yearly Susceptibility Test Information Collection antimicrobial susceptibility program in Spain: a 5-year analysis. Diagn Microbiol Infect Dis 2007;57(2):195-200.

27. Sader H.S., Fritscche T.R., Jones R.N. Potency and spectrum trends for cefepime tested against 65,746 clinical bacterial isolates collected in North American medical centers: results from the SENTRY Antimicrobial Surveillance Program (1998-2003). Diagn Microbiol Infect Dis 2005;52(3):265-73.

28. Turner P.J. Susceptibility of meropenem and comparators tested against 30,634 Enterobacteriaceae isolated in the MYSTIC programme (1997-2003). Diagn Microbiol Infect Dis 2004;50(4):291-3. 